\section{ANAESTHESIA IN PAEDIATRIC BRONCHOSCOPY}

\author{
KHADGARAY R.N.
}

Address for Correspondence:-

Dr. R.N. Khadgaray

Department of Anaesthesiology

U.C.M.S. Teaching Hospital, Ranigaon, Bhairahawa, Nepal.

General anaesthesia for emergency bronchoscopy is Frought with dangers especially in paediatric age group where inhalation of foreign bodies are common especially where respiratory obstruction is present and management becomes perilous.

\section{CASE REPORT}

A 4 year child was brought who was febrile and dyspnoeic. Emergency bronchoscopy under general anaesthesia was contemplated. Preanaesthetic Assessment revealed a well nourished toxic looking child with a temperature of $39.1^{\circ} \mathrm{C}$ and Pulse rate of $136 /$ min and respiratory rate was 40/min with a mild cyanosis. On auscultation wheezing sounds were heard over right middle lobe with absent breath sounds over right lower lobe. X-Ray revealed collapsed right lower lobe, mediastinal shift to the right side and compensatory emphysema of left lung. After premedication with Hyoscine and dexamethasone induction was carried out with midazolam. Patient was allowed to breathe $50 \%$ mixture of nitrous oxide and oxygen .Soon after the child went to sleep $60 \mathrm{mg}$ of $1 \%$ lignocaine was administered i.v. After 3 min surgeon was allowed to proceed with bronchoscopy.

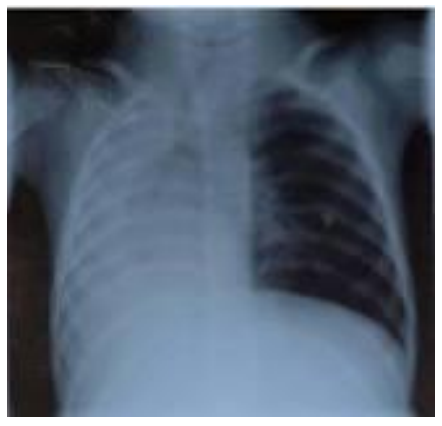

Analgesia was maintained with intravenous Ketamine hydrochloride while the child was allowed to breathe the anaesthetic gas mixture delivered through the side tube of bronchoscope.

The procedure lasted about 25 minutes and multiple small pieces of ground nut were removed. At the end of operation Patient was oxygenated and trachea aspirated. Patient had no memory of the operative procedure and emergence phenomenon was not observed. Post operatively he was managed with bronchodilators, antibiotics and decongestant and physiotherapy. Lungs became clinically and radiologically clear post operatively. Conventional general anaesthesia with thiopentone, inhalational agent and muscle relaxant is considered and dangerous. Midazolam when administered intravenously causes sedation and mild relaxation of skeletal muscle, 
anterograde amnesia and minimal depression of cardio-respiratory system.

Intravenous lignocaine causes suppression of pharyngeal and laryngeal reflexes without significant depression of respiration central sedation and adequate analgesia. In this case good relaxation of jaws was provided by lignocaine and midazolam. Ketamine provided good analgesia without depression of respiration. Cardiovascular stability was well maintained because of prior administration of Midazolam. Emergence phenomenons were not encountered. The technique of analgesia appears to be adequate for such procedure and potent anaesthetic which can adversely affect cardiovascular system are avoided.

\section{REFERENCES}

1. Millers Anaesthesia-9 $9^{\text {th }}$ edition 1853.

2. Paul a Baresn, Text book Anaesthesia-- $6^{\text {th }}$ edition 1655

3. Moregan, Clinical Anaesthesia-4 ${ }^{\text {th }}$ edition 1606. 